\title{
Perspectivas da Investigação sobre Determinantes Sociais em Câncer
}

\author{
I Victor Wünsch Filho, ${ }^{2}$ José Leopoldo Ferreira Antunes, ${ }^{3}$ Antonio Fernando Boing, \\ ${ }^{4}$ Ricardo Luiz Lorenzi I
}

Resumo: As diferenças socioeconômicas têm reflexos no perfil epidemiológico de câncer, no que diz respeito a incidência, mortalidade, sobrevida e qualidade de vida após o diagnóstico. Neste artigo examinam-se as disparidades da ocorrência de câncer na população brasileira e sintetizam-se evidências das investigações sobre determinantes sociais em câncer. Foram considerados os principais fatores que modulam a influência das condições socioeconômicas na ocorrência do câncer, como tabagismo, consumo de álcool, hábitos alimentares e obesidade, ocupação e acesso aos serviços de saúde. Modificações nas condições sociais dependem de mudanças estruturais na sociedade, a exemplo de melhorias do nível educacional; no entanto, investigaçōes epidemiológicas bem conduzidas podem contribuir para o planejamento de intervençôes visando a reduzir o impacto dos determinantes sociais em câncer. Esses estudos devem prover estratégias para promoção da qualidade das informaçōes de incidência e mortalidade; realização periódica de inquéritos populacionais sobre prevalência de fatores de risco para câncer; desenvolver desenhos epidemiológicos mais eficientes para avaliar o efeito de fatores etiológicos em câncer e suas relaçôes com o status social; análise de programas de rastreamento para tumores passíveis de detecção precoce; e avaliaçōes do acesso da população ao diagnóstico e tratamento. Essas pesquisas devem contemplar populações em distintas regiões do mundo, em particular aquelas vivendo em regióes marginalizadas da dinâmica do atual sistema econômico global.

\footnotetext{
> Palavras-chave: câncer; condições socioeconômicas; desigualdades em saúde.
}

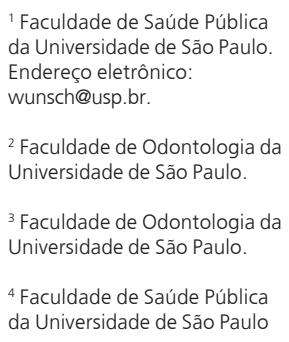

${ }^{4}$ Faculdade de Saúde Pública da Universidade de São Paulo 


\section{Introdução}

A associação entre níveis socioeconômicos da população e a incidência e mortalidade por câncer envolve múltiplos aspectos. Distintos padrões culturais entre diferentes classes sociais têm influência nesta relação, e são contínuas as mudanças no tempo e espaço de estilos de vida e exposiçõos de risco para câncer entre classes sociais. Nos países afluentes, tabagismo e obesidade, importantes fatores de risco para câncer, apresentam atualmente prevalências mais elevadas em populaçôes de estratos socioeconômicos inferiores, invertendo um padrão anterior (KAWACHI; KROENKE, 2006; LYNGE et al., 2006). A localização anatômica do tumor é outra variável relevante na análise dos determinantes sociais em câncer. Notadamente, tumores de colo de útero e de cabeça e pescoço apresentam maior incidência nos estratos populacionais com piores condições sociais. Em contraposição, tumores de mama e cólon são mais expressivos nos estratos diferenciados da sociedade (WÜNSCH FILHO; MONCAU, 2002; PALACIOMEJÍA et al., 2003; CONWAY et al., 2008). Tais tendências são dinâmicas e estão em constante transformação, que não ocorrem ao azar, mas no sentido de conferir maior carga da doença entre os mais pobres. Exemplo é a constatação de aumentos sucessivos das taxas de mortalidade por câncer de mama nas coortes de mulheres nascidas após 1950 e residentes nas áreas empobrecidas da cidade de São Paulo (PRADO, 2002).

As desigualdades na ocorrência de câncer são relevantes e devem ser preocupação dos epidemiologistas neste momento histórico em que as discrepâncias na saúde tendem a aumentar de magnitude pelo processo de globalização, crescente urbanização e precariedade no trabalho (BARTEN et al., 2008). O estudo das desigualdades em saúde permite reconhecer possíveis mecanismos causais das doenças e identificar oportunidades de intervenções preventivas com alto potencial de efetividade (KAPLAN, 1998). Por outro lado, é imperativo reduzir as iniqüidades em saúde, pois são eticamente inaceitáveis, são evitáveis ou remediáveis e afetam a todos na população.

Neste artigo serão examinadas as disparidades da ocorrência de câncer no Brasil. Também, serão discutidas as limitações metodológicas dos estudos que buscam relacionar as determinaçôes sociais em câncer, particularmente no referente à construção da cadeia causal nos desfechos de câncer. Serão revisados os fatores de risco conhecidos para câncer em relação à prevalência nos distintos estratos sociais 
da população. Finalmente, serão discutidos os desafios da investigação dos determinantes sociais em câncer.

\section{Desigualdades na incidência, mortalidade e sobrevida por câncer no Brasil}

Informações limitadas e de baixa qualidade de agravos à saúde provenientes de populações vivendo em situações de precariedade social são a principal dificuldade para a comparação da incidência, mortalidade e sobrevida por câncer entre populações do mundo ou entre regióes de um mesmo país.

\section{Incidência}

A avaliação de diferenças de incidência por câncer entre populaçôes requer dados de registros de câncer de base populacional. No volume IX do Cancer in Five Continents - última versão da tradicional publicação da Agência Internacional de Pesquisas em Câncer -, observa-se que a maioria dos dados provém de países desenvolvidos, com informaçôes menos abrangentes para países da África e América do Sul (CURADO et al., 2007). Historicamente, a participação do Brasil nas diferentes versões daquela publicação esteve restrita aos dados de poucas cidades. No volume VIII participaram Campinas e Goiânia (PARKIN et al., 2002); no volume IX, apenas os registros de Brasília, Cuiabá, Goiânia e São Paulo atingiram níveis mínimos de qualidade dos dados para serem incluídos naquela publicação (CURADO et al., 2007).

O Instituto Nacional do Câncer (INCA) realiza estimativas de incidência de câncer no país, utilizando, além dos quatro registros incluídos no volume IX do Cancer in Five Continents, informaçôes de outros 15 registros de câncer de base populacional (BRASIL, 2007). Embora as estimativas de incidência divulgadas pelo INCA sejam taxas brutas e haja grandes diferenças na qualidade das informaçōes geradas por esses registros, os dados indicam, grosso modo, marcantes contrastes na incidência de câncer. As taxas de incidência do conjunto de tumores são cerca de duas vezes mais elevadas no Sudeste e Sul, regiōes com melhores indicadores socioeconômicos, do que no Norte e Nordeste, regiôes mais afetadas pela privação material. A região Centro-Oeste apresenta perfil intermediário de incidência.

As explicações para tais diferenças regionais não são óbvias. As neoplasias mais estritamente relacionadas ao tabaco (pulmão, cavidade oral, esôfago) apresentam 
incidência mais alta no Centro-Oeste, Sudeste e Sul, regiões mais industrializadas e com maior prevalência de tabagismo (INCA, 2004). As taxas de incidência do câncer de mama feminino também são maiores nas regiōes Sudeste e Sul. Por outro lado, a incidência do câncer de colo de útero destaca-se na região Norte, em relação às demais. Este padrão contrastante das incidências do câncer de mama e colo de útero com relação a populações mais ou menos afluentes apresenta correspondência com o observado na comparação de incidências desses tumores entre países (FAGGIANO et al., 1997).

\section{Mortalidade}

A interpretação dos dados de mortalidade por câncer é ainda mais difícil, pois além dos fatores relacionados à incidência, outros aspectos precisam ser considerados, como os diferenciais de acesso a tratamentos efetivos, variações de sobrevida e, também, os riscos competitivos de morte.

$\mathrm{O}$ indicador que traduz mais diretamente a qualidade das estatísticas de mortalidade de um país ou região é a proporção de mortes atribuídas às causas mal definidas (SILVI, 2003; MATHERS et al., 2005). No Brasil, em 2003, 13,3\% dos óbitos tiveram a causa básica classificada como mal definida, com grande discrepância entre as regiōes: maiores taxas no Nordeste (25,9\%) e Norte $(21,2 \%)$ e menores no Centro-Oeste (5,7\%), Sul (6,7\%) e Sudeste (8,9\%). Observou-se o mesmo padrão em relação às taxas de óbitos que não receberam assistência médica (SANTO, 2008). Esses indicadores interferem drasticamente na interpretação dos estudos de mortalidade no país.

A confiabilidade dos dados de diagnóstico nos atestados de óbito está relacionada a idade, etnia, tempo de sobrevida, lugar de residência e status socioeconômico. $\mathrm{Na}$ comparação com outros países, os dados de mortalidade do Brasil são considerados de qualidade intermediária (MATHERS et al., 2005). Entretanto, o câncer é considerado como uma das causas de morte com melhor registro nos atestados de óbito (PERCY et al., 1981), o que permite assumir a mortalidade por câncer como informação consistente relativamente a outras doenças, mesmo para a população brasileira.

A mortalidade por câncer também mostra um padrão marcantemente heterogêneo entre as regiôes do país. Em termos absolutos, o risco de morrer por câncer é maior no Sul e Sudeste, porém as taxas de mortalidade para o conjunto de 
todos os tipos de câncer apresentam tendência decrescente nas duas regiōes, tanto entre homens como entre mulheres. Em contraposição, embora as taxas sejam mais baixas nas regiōes Centro-Oeste, Nordeste e Norte, a tendência da mortalidade por câncer (todos os tipos de tumores) nestas regiōes são ascendentes (WÜNSCH FILHO; MONCAU, 2002).

Estudo na cidade de São Paulo mostrou a dinâmica relação entre condições socioeconômicas, espaço geográfico e mortalidade por neoplasias femininas (PRADO, 2002). Nos territórios mais ricos da cidade, a mortalidade por câncer de mama manteve-se estável de 1985 a 1999. Ao contrário, nas áreas periféricas, a mortalidade aumentou sistematicamente no período. Por outro lado, a mortalidade por câncer de colo de útero decresceu de forma distinta nos territórios analisados, com tendência menos expressiva nos bairros com piores condiçōes de vida e saúde. Em síntese, as populaçôes femininas das regiões menos afluentes da cidade tendem a atingir o padrão da mortalidade por câncer de mama das populações femininas nos territórios geográficos com maior afluxo econômico, porém a queda da mortalidade por câncer de colo de útero é bem mais lenta nas áreas carentes da cidade.

\section{Sobrevida}

Paralelamente às informações sobre incidência e mortalidade, a análise de sobrevida de pacientes com câncer permite avaliar aspectos do controle de câncer, como as estratégias de detecção precoce e o acesso a serviços de saúde para diagnóstico e tratamento. O estudo de sobrevida de pacientes com câncer é útil para avaliações comparativas - por exemplo, para examinar diferenças no tempo e entre subgrupos de idade, sexo, etnia e status socioeconômico (SANKARANARAYANAN et al., 1998).

Baixos níveis socioeconômicos são consistentemente relacionados a um pior prognóstico após o diagnóstico de câncer. Mesmo para os tumores mais incidentes nos estratos populacionais de melhor condição social, como câncer de mama e melanoma, a sobrevida apresenta relação inversa.

Estudo comparando a sobrevida de pacientes atendidos por câncer de cabeça e pescoço em dois centros especializados de atenção ao câncer, em Nova York e São Paulo, revelou maior sobrevida média no centro americano que no centro paulista. Como os dois centros utilizam abordagens terapêuticas semelhantes, explicou-se a diferença de sobrevida pelo diagnóstico de tumores em estádio mais avançado em 
São Paulo. Este fato pode ser decorrência de uma combinação de fatores, entre os quais se destacam o nível educacional dos pacientes e a percepção dos primeiros sintomas, além de limitações no acesso à atenção médica e o retardo na confirmação diagnóstica (CARVALHO et al., 2004).

\section{Limitações metodológicas na mensuração da exposição e na avaliação das taxas de câncer}

Em muitas circunstâncias, as evidências das relações entre condições socioeconômicas e a ocorrência de câncer não podem ser assumidas como conclusivas, mas permitem inferências sobre fatores relacionados ao estilo de vida moderno, que podem modificar o impacto de exposiçôes ambientais e/ou a suscetibilidade dos indivíduos. Por outro lado, as dificuldades das pesquisas para estabelecer estas associaçõos de forma mais consistente dependem, entre outros, de dois fatores principais: informações insuficientes ou precárias, situação vigente com freqüência nos países em desenvolvimento; e fragilidades metodológicas intrínsecas dos estudos.

O caso da leucemia infantil é emblemático. Tem sido sugerido que a leucemia em crianças seria uma das raras exceções em câncer, cuja incidência seria maior em estratos de melhor condição socioeconômica. Em abrangente revisão, Poole et al. (2006) sumarizaram 47 estudos sobre a associação entre status socioeconômico e leucemia em crianças. Foi encontrada associação positiva em cerca de metade dos estudos e negativa na outra metade. Os autores mostraram que a direção da associação diferia de acordo com o desenho do estudo epidemiológico e com a mensuração utilizada para avaliar o status socioeconômico. Estudos com base em registros de câncer e prontuários hospitalares geralmente produziram associações positivas, enquanto estudos caso-controle, com informações obtidas em entrevistas, associações negativas. Quando o nível educacional dos pais foi considerado, em geral os resultados se mostraram predominantemente negativos, isto é, maiores taxas nos grupos com status socioeconômico mais baixo. Entretanto, a classe ocupacional dos pais foi em geral associada positivamente com o estrato socioeconômico, isto é, maiores taxas nos grupos com níveis mais altos de status econômico. Também se observou certa heterogeneidade temporal, com estudos antigos gerando associações positivas e estudos recentes, associações negativas. $\mathrm{O}$ viés de seleção relacionado ao status socioeconômico também pode ser importante 
fator explicativo dos resultados heterogêneos encontrados. Os estudos caso-controle incluíram a maioria dos casos, mas em geral somente subgrupos de controles, resultando em possível super-representação de controles oriundos de níveis mais altos de status socioeconômico. Em contraste, estudos com base em registros de câncer podem ter sofrido tendência de sub-representação de casos das classes sociais mais baixas (KUEHNI; ZWAHIEN, 2006; POOLE et al., 2006).

Estudos epidemiológicos têm mostrado que, mesmo após ajuste para fatores de risco reconhecidos, como tabagismo e consumo de álcool, ainda há um efeito residual das condições sociais sobre o risco de câncer (CONWAY, 2008). Entretanto, esse fato pode ser decorrência da influência de outros fatores mediadores das condições sociais em câncer, não completamente explorados no contexto de um determinado estudo. Nos modelos hierárquicos, utilizados crescentemente na análise epidemiológica, as condições socioeconômicas são incluídas como determinantes distais de doenças e condições de saúde, enquanto variáveis comportamentais e de estilo de vida como proximais (VICTORA et al., 1997). Tal abordagem analítica parece ser uma forma mais coerente e efetiva de avaliar a implicação de diferentes variáveis dentro de um modelo de cadeia causal.

A seguir serão revisados alguns dos principais fatores de risco intervenientes na relação entre determinantes sociais e câncer, considerando-se estudos que buscaram avaliar as variações da prevalência de acordo com os estratos sociais nas populações.

\section{Desigualdades sociais e câncer: fatores intervenientes}

Nas últimas décadas, foram obtidos relevantes progressos na compreensão dos fatores que afetam a morbi-mortalidade por câncer, sua prevenção, detecção, tratamento e da qualidade de vida dos sobreviventes. Todavia, os benefícios decorrentes desse conhecimento não foram distribuídos de modo homogêneo pelas populaçôes (LOPEZ et al., 2004). A carga de doença nos grupos de níveis socioeconômicos mais baixos pode ser inferida pela maior incidência de câncer em geral; pelo diagnóstico tardio de neoplasias passíveis de detecção em estágios iniciais por meio de rastreamento; pelas dificuldades de acesso ao diagnóstico e tratamento adequado; pelas incapacidades adquiridas em decorrência da doença; pelo maior risco de óbito por tipos de câncer potencialmente curáveis; pelo não-tratamento para o controle da dor e insuficiência de outros cuidados paliativos. 
A suscetibilidade ao câncer é determinada pela interação de múltiplos genes, portanto, o câncer caracteriza-se como uma doença genética. Entretanto, a ocorrência da doença é fortemente determinada por fatores como dieta, tabagismo, agentes infecciosos e exposições ambientais e ocupacionais a agentes cancerígenos (PHAROAH, 2003; UAUY; SOLOMONS 2005). A prevalência desses fatores nas populações está estritamente conectada às desigualdades sociais. A seguir alguns desses fatores serão examinados nas suas inter-relações com os distintos estratos socioeconômicos de populaçôes e que podem determinar as disparidades observadas na ocorrência de neoplasias. Também será discutido o acesso aos serviços de saúde e à detecção precoce de câncer, cujas disparidades podem influenciar de forma dramática a sobrevida e a qualidade de vida dos pacientes com câncer oriundos das parcelas mais pobres da população.

\section{Tabagismo}

O consumo de tabaco é considerado o principal fator de risco isolado para câncer. Medidas restritivas de comercialização e consumo do tabaco nos países ricos levaram a indústria do tabaco a direcionar seus esforços de venda para países mais pobres onde havia perspectiva de crescimento do consumo. Com isso, no final da década de 90, dos cerca de 100 mil jovens que se iniciavam no tabagismo a cada dia, 80\% residiam em países em desenvolvimento (JHA; CHALOUPKA, 1999).

$\mathrm{Na}$ Nova Zelândia, Whitlock et al. (1997) identificaram gradiente socioeconômico no consumo de tabaco. A prevalência foi $12 \%$ entre pessoas com educação universitária e 35\% entre aquelas com menos de dois anos de estudo. A estratificação da população por faixas de renda e ocupação revelou consumo maior, duas a três vezes, nos grupos com piores condições sociais. Além disso, baixa renda, ocupação em atividades com menor qualificação e restrita escolaridade foram fatores preditivos de baixas taxas de abandono do tabagismo e de maior quantidade de cigarros fumados. A correlação do consumo de tabaco em grupos de menor renda, pior nível educacional e exercendo ocupações precárias é um resultado que se repete monotonamente nas pesquisas realizadas em vários países (SOTERIADES; DIFRANZA, 2003; FEDERICO et al., 2004; VILLALBI et al., 2004; FUKUDA et al., 2005; WEN et al., 2005; HARMAN et al., 2006; KHANG; CHO, 2006).

Em 1987, Lolio et al. (1993) investigaram em Araraquara, no Estado de São Paulo, as diferenças na prevalência de fumantes entre estratos socioeconômicos. 
Em homens analfabetos ou com menos de quatro anos de estudo, a prevalência de tabagismo atingiu $48,6 \%$, enquanto nos indivíduos com curso superior completo a prevalência foi $35,7 \%$. Tendência análoga, mas com porcentagens inferiores, foi observada entre as mulheres. Nos anos 1990, em Porto Alegre, verificou-se maior prevalência de tabagismo entre homens que recebiam abaixo de dois salários mínimos, que tinham menos de cinco anos de estudo e exerciam ocupações menos qualificadas (MOREIRA et al., 1995). Dados equivalentes foram anotados em Belo Horizonte (LIMA-COSTA, 2004). Szwarcwald et al. (2004) analisaram amostra representativa da população brasileira e estimaram em $30 \%$ a porcentagem de homens fumantes entre os que dispunham menos de quatro bens no domicílio (televisão, geladeira, microondas, máquina de lavar roupa, máquina de lavar louça, microcomputador, aparelho de som, telefone fixo, telefone celular ou carro); entre os que possuíam mais de oito desses bens, a proporção de fumantes foi $21 \% \mathrm{Na}$ cidade de São Paulo, maior prevalência de tabagismo foi constatada entre indivíduos com menor nível educacional (CÉSAR, 2005).

Inquérito sobre fatores de risco para doenças não-transmissíveis realizado em 16 capitais do país mostrou prevalências mais altas de tabagismo nas cidades brasileiras mais industrializadas do Sudeste e Sul, em ambos os sexos (INCA, 2004). Em 1989, a prevalência atingia 33,1\% dos indivíduos de ambos os sexos, com 15 ou mais anos de idade (BRASIL, 1990). Atualmente, Porto Alegre é a capital brasileira com mais alta prevalência $(25,2 \%)$ de tabagismo (INCA, 2004; IGLESIAS et al., 2007). Essa tendência é a principal explanação causal para o declínio da mortalidade por tumores pulmonares no país entre homens com menos de 70 anos de idade. Todavia, a tendência é ascendente nas mulheres, em função do aumento da prevalência do tabagismo entre as mulheres em décadas recentes (MALTA et al., 2007).

Os mais altos níveis de prevalência de tabagismo, em ambos os sexos, bem como a ocorrência das mais altas taxas de incidência de câncer de pulmão no país, são constatados em Porto Alegre. O Brasil é o segundo maior produtor mundial de tabaco em folha (14,1\% da produção mundial), atrás somente da China. O Estado do Rio Grande do Sul é responsável por cerca de 50\% da produção nacional. A cultura é típica de pequenas propriedades e a maior parte da produção localizase no entorno de indústrias de transformação e beneficiamento (SEPLAG, 2002). Esta característica econômica pode ter definido intrincadas relações entre produção 
agrícola e industrial, cultura, submissão a determinados hábitos e ações políticas (lobbies de produtores agrícolas e da indústria do tabaco) que podem ter influenciado o consumo mais elevado de tabaco naquele estado.

\section{Consumo de bebidas alcoólicas}

O consumo de bebidas alcoólicas é variável no tempo e nas diferentes sociedades, tanto em termos de quantidade como de qualidade. Aspectos culturais interagem tanto na definição da dieta quanto no padrão de consumo de álcool. Nos estratos privilegiados, o consumo de vinho é atualmente maior que há algumas décadas, quando predominava o consumo de destilados. Em geral, esse aumento de consumo de vinho promove mudanças na dieta, com uma composição mais rica em fatores protetores para câncer. $\mathrm{O}$ consumo excessivo de bebidas alcoólicas tem sido relacionado com a maior ocorrência de alguns tumores. Entretanto, avaliar o efeito etiológico isolado do álcool sobre câncer é difícil, por sua ligação com outros fatores potencialmente carcinogênicos, em particular o tabagismo. Além disso, o abuso no consumo de álcool conduz a uma ruptura social com isolamento do indivíduo e dificultando a obtenção de informações confiáveis (MARSHALL; FREUDENHEIN, 2006).

$\mathrm{Na}$ Comunidade Européia, o consumo excessivo de álcool foi mais freqüente entre pessoas com menor escolaridade (BLOOMFIELD et al., 2006). Em Roterdam, Holanda, menores taxas de abstinência foram observadas entre indivíduos com maior escolaridade e, entre os que ingeriam bebidas alcoólicas, o nível de consumo era maior entre pessoas menos escolarizadas (VAN OERS et al., 1999). Distintas regióes dos Estados Unidos apresentaram maior prevalência de consumo de álcool entre indivíduos de menor escolaridade (GALEA et al., 2007). $\mathrm{Na}$ Índia, também foi encontrado gradiente negativo entre nível socioeconômico e consumo de bebidas alcoólicas (SUBRAMANIAN et al., 2005).

Inquérito populacional identificou padrões heterogêneos de consumo de álcool entre as regiōes brasileiras, com maiores porcentagens de bebedores no Sul e Sudeste que no Norte e Nordeste (INCA, 2004). Em Porto Alegre, inquérito populacional identificou maiores porcentagens de alcoolismo entre negros e pardos, indivíduos com menor escolaridade e residentes em domicílios com alto índice de aglomeração (MOREIRA et al., 1996). Resultados mais recentes foram similares em outras cidades do Rio Grande do Sul (MENDONZA-SASSI; BERIA, 2003; COSTA et 
al., 2004). Também na cidade de São Paulo, maior consumo de bebidas alcoólicas foi observado entre indivíduos com menor nível educacional (CÉSAR, 2005). Entretanto, os resultados da associação entre condição socioeconômica e consumo de álcool são conflitantes. Em Salvador, descreveu-se maior prevalência de consumo entre pessoas com melhores ocupaçōes (ALMEIDA-FILHO et al., 2004). Estudo com base em amostra nacional identificou que, dentre pessoas com oito ou mais bens no domićlio, 19\% consumiram cinco ou mais doses de bebida alcoólica na semana anterior à entrevista, enquanto dentre os que possuíam de zero a três bens, a proporção foi 11,8\% (SZWARCWALD et al., 2004).

\section{Dieta, obesidade e atividade física}

A dieta reflete os fluxos da economia, mudanças nas alianças geopolíticas e de comércio, e define os valores de uma sociedade. Para os indivíduos, a dieta reflete o status e a condição favorável ou desfavorável de saúde. O consumo de frutas e vegetais tem sido descrito como fator de proteção na ocorrência de diferentes tipos de câncer. Dieta inadequada e sedentarismo induzem à obesidade. Esses fatores, isoladamente ou em conjunto, influenciam a incidência de câncer.

Em Israel, os grupos populacionais com melhores condições econômicas consumiam mais frutas e vegetais, além de proteínas, fibras, ferro, cálcio, magnésio e vitaminas (SHAHAR et al., 2005). No Canadá, escolares de regiões mais ricas consumiam mais vegetais, frutas e fibras (MINAKER et al., 2006). Na Holanda, adultos de nível educacional mais elevado e exercendo ocupaçôes mais bem remuneradas consumiam menos gorduras e mais vegetais, queijos e fibras (HULSHOF et al., 2003).

Com base nos dados da Pesquisa Mundial da Saúde (World Health Survey), conduzida pela Organização Mundial da Saúde, Jaime \& Monteiro (2005) identificaram que, diariamente, $41 \%$ dos adultos brasileiros consomem frutas e $30 \%$, hortaliças. Observaram ainda a influência dos determinantes sociais sobre o padrão alimentar, com correlação positiva entre o consumo diário de frutas e o número de anos de estudo dos indivíduos. Informações geradas pela Pesquisa de Orçamento Familiar 2002-2003 mostraram que a participação relativa de frutas e sucos naturais no total de calorias consumidas por brasileiros com renda superior a cinco salários mínimos foi 5,5 vezes mais elevada do que entre pessoas com até $25 \%$ de um salário mínimo. O grupo com renda mais alta apresentou também 
consumo mais elevado de verduras, legumes, óleos e gorduras vegetais, gordura animal, leite e derivados (LEVY-COSTA et al., 2005).

A obesidade afeta mais de 300 milhões de adultos no mundo. Nos países desenvolvidos, o excesso de peso corporal é o quinto fator de risco mais importante para doenças (BLEICH et al., 2008). O aumento do índice de massa corpórea (IMC) aumenta o risco de diabetes tipo 2, hipertensão arterial sistêmica e outras doenças cardiovasculares, bem como de tumores malignos do cólon, mama feminina (pós-menopausa), endométrio, esôfago (adenocarcinoma), tiróide (em mulheres) e próstata (UAUY; SOLOMONS 2005; BALLARD-BARBASH et al., 2006).

A epidemia mundial de obesidade é crescente e com persistência de gradientes sociais. É um problema global com distribuição desigual entre e dentro de países. Nas sociedades mais ricas, o excesso de peso é mais comum nos grupos com desvantagens sociais, mas o inverso é observado nos países em desenvolvimento (MONTEIRO et al., 2004; FRIEL et al., 2007).

As populações latino-americanas urbanizaram-se nas últimas décadas e, como conseqüência, o consumo de alimentos com alta densidade de energia (gorduras e açúcar) aumentou acentuadamente e a atividade física decaiu, com tendência crescente ao sedentarismo (UAUY; MONTEIRO, 2004). No Brasil, de acordo com o mais recente inquérito populacional, cerca de $12 \%$ da população adulta é obesa - IMC igual ou maior a $30 \mathrm{~kg} / \mathrm{m}^{2}$ (INCA, 2004). Nas regióes CentroOeste, Sudeste e Sul, a prevalência foi maior nas pessoas de menor escolaridade; o inverso foi observado nas regiōes Nordeste e Norte. Na década de 90, pesquisa de base nacional mostrou aumento de obesidade em todos os níveis de escolarização. Entretanto, desde então, o aumento restringiu-se aos grupos menos escolarizados, estabilizando-se ou mesmo decrescendo em mulheres com escolaridade média ou elevada (MONTEIRO et al., 2007).

O aumento da obesidade é conseqüência de um sistema moldado pela organização das sociedades nos planos mundial, nacional e local. Grupos populacionais de estratos mais baixos são afetados pelo ambiente, com poucas opções nas regiões empobrecidas em que vivem de alimentação saudável e de atividades físicas e lazer, além de deficiências de transporte público (FRIEL et al., 2007). A discussão da obesidade implica considerar aspectos como uso e ocupação do território urbano, trabalho, atividade física e oportunidades de lazer, tipo de dieta e as conexões destas variáveis com o consumo de álcool e tabaco. 


\section{Ocupação}

A ocupação é dimensão fundamental da condição socioeconômica. Ao lado de renda e escolaridade, a ocupação forma o tripé básico de variáveis que permite avaliar a situação socioeconômica dos indivíduos. Trabalhadores inseridos em atividades manuais ou aqueles com pouca qualificação profissional estão submetidos a maiores riscos de exposição a substâncias químicas, fumaça de diesel, tintas e corantes, e outros agentes cancerígenos (BOFFETTA et al., 1997). Esses trabalhadores também apresentam baixo consumo de frutas e verduras, com deficiências de micronutrientes. É reconhecido que deficiências de vitaminas podem alterar a biotransformação de agentes tóxicos no organismo e, portanto, sua toxicidade (PEARCE et al., 1994). Assim, estes indivíduos apresentam a situação cumulativa de vulnerabilidade orgânica e de exposição, por vezes a níveis bastante altos, a substâncias cancerígenas nos locais de trabalho.

Exposiçôes mais intensas a agentes nocivos nos ambientes de trabalho são observadas nos países em desenvolvimento (CHRISTIANI; WANG, 2003). Esta situação é atribuída à transferência seletiva de indústrias nocivas e que utilizam tecnologias obsoletas para os países do capitalismo periférico, num contexto de baixo nível de informação dos riscos inerentes a essas atividades. Apesar de esta condição ter sido alvo de disposição normativa no início desta década, pela Organização para Colaboração Econômica e Desenvolvimento (OECD, 2003), o processo parece ainda estar em curso.

O trabalho modula a exposição a agentes cancerígenos ambientais mais gerais. Estudo prospectivo europeu com mais de 200.000 participantes identificou risco aumentado de câncer de pulmão em profissões tabaco-relacionadas (VEGLIA et al., 2007). Trabalhadoras do sexo estão mais vulneráveis à infecção pelo HPV (JUÁREZ-FIGUEROA et al., 2001). Além da natureza do trabalho, outros aspectos foram relacionados a diferenciais na ocorrência de câncer como decorrência da condição de emprego. Na Finlândia, mostrou-se associação entre trabalho de vínculo precário e aumento de mortalidade por formas de câncer relacionadas ao tabaco (KIVIMÄKI et al., 2003).

O trabalho infantil, decorrente das condições de pobreza e da falta de controle social adequado, é uma outra face das estritas conexôes entre determinantes sociais e condições de trabalho na gênese do câncer. Além de eticamente condenável, o trabalho infantil submete as crianças a exposições precoces a agentes tóxicos. Considerando- 
se o período de latência, a probabilidade de indivíduos expostos a substâncias cancerígenas na infância virem a desenvolver algum tipo de câncer é potencialmente maior do que naqueles com início de exposição similar na vida adulta.

Outra dimensão de análise dos determinantes ocupacionais do câncer referese à classe social. Em coorte com 20.000 indivíduos identificou-se forte gradiente socioeconômico na incidência de tumores relacionados ao tabaco e ao álcool entre trabalhadores franceses exercendo ocupações manuais e atividades de escritório (MELCHIOR et al., 2004). Menvielle et al. (2004) observaram associação entre câncer de laringe e hipofaringe e trabalho manual, utilizando categorias de classe e de mobilidade social, construídas com base em títulos de ocupações e atividades econômicas.

\section{Uso dos serviços de saúde}

Além de maior exposição aos principais fatores de risco para câncer, os indivíduos dos estratos sociais mais baixos têm acesso restrito aos serviços de saúde. Em geral, essa dificuldade implica demora do diagnóstico. Como conseqüência, a detecção tardia de tumores traduz-se em pior prognóstico e intervençôes terapêuticas extensas e mutiladoras.

Na Austrália, Nova Zelândia, Canadá, Estados Unidos e Reino Unido, identificaram-se maiores dificuldades para as pessoas de baixa renda em obter consultas com médicos especialistas (SCHOEN; DOTY, 2004). Em países da Europa Ocidental, o acesso a cuidados de saúde não é igualitário quando o paciente necessita financiar parte dos serviços prestados (LOSTAO et al., 2007).

No Brasil, Travassos et al. (2002) analisaram o uso dos serviços de saúde estratificando a população brasileira em dois grupos: pessoas com algum tipo de restrição das atividades rotineiras em função de problemas de saúde e pessoas sem qualquer restrição. Nos dois grupos, houve maior freqüência de utilização dos serviços de saúde por parte das pessoas brancas e com maior escolaridade e renda.

\section{Detecção precoce de câncer}

A detecção precoce é um dos principais fatores a interferir na sobrevida de pacientes com câncer. Para três tipos de câncer, há evidências de que programas de rastreamento podem ser úteis e efetivos na detecção precoce: cólon, mama e colo de útero (ELUF NETO; WÜNSCH FILHO, 2004). Recentemente, assinalaram- 
se evidências de efetividade do rastreamento de tumores de cavidade bucal em grupos de alto risco (SANKARANARAYANAN et al., 2005).

Do ponto de vista prático, propostas de rastreamento para tumores de cólon são de implementação mais difícil, pois exigem a avaliação de sangue oculto nas fezes. Para os tumores bucais, o conhecimento científico acumulado indica a necessidade prévia de identificar os grupos de população com maior risco, o que restringe a abrangência das ações de prevenção secundária. Para os tumores essencialmente femininos, há necessidade de programas de rastreamento de base nacional. No Brasil, não há programas de rastreamento estruturados para esses tumores; o que existe é uma forma de rastreamento informal e espontâneo por parte das mulheres, estratégia mais bem utilizada por aquelas de maior poder aquisitivo e com acesso privilegiado a informação e serviços de saúde.

A cobertura de rastreamento para o câncer de colo uterino apresenta grandes desigualdades entre países (GAKIDOU et al., 2008). No Brasil, inquérito populacional para fatores de risco realizado em 2003 avaliou o percentual de mulheres que espontaneamente procuraram realizar exames de rastreamento para tumores de colo de útero e mama (INCA, 2004). A proporção de mulheres que relataram ter realizado pelo menos um exame de Papanicolaou nos últimos três anos foi superior a 80\% nas capitais estudadas, com exceção de João Pessoa, Belém e Aracaju. Em todas as cidades estudadas, à exceção de Aracaju, o relato da realização do exame Papanicolaou esteve associado positivamente ao grau de escolaridade. No tocante à realização de mamografia nos dois anos anteriores à entrevista, as capitais da Região Norte apresentaram os menores percentuais de respostas positivas: Belém 36,8\% e Manaus 42,3\%. Mas também em Fortaleza, Natal, Aracaju, Distrito Federal e Rio de Janeiro menos da metade das mulheres realizou este exame nos dois anos anteriores à entrevista. Em todas as capitais, exceto Campo Grande, a realização de mamografia correlacionou-se positivamente com escolaridade.

\section{Perspectivas para a investigação dos determinantes sociais em câncer}

A prevenção do câncer depende primeiramente da compreensão de sua etiologia. A complexidade da etiologia em câncer envolve fatores de risco em diferentes níveis, do social e econômico ao genético e molecular. Portanto, a moderna pesquisa epidemiológica sobre etiologia do câncer não está limitada a simplesmente definir 
a exposição dos indivíduos a fatores de risco como tabagismo ou consumo de bebidas alcoólicas. A prevenção do câncer comporta ainda considerar a detecção precoce e o pronto diagnóstico e tratamento. No contexto abrangemte de prevenção, a identificação dos grupos populacionais mais vulneráveis é fundamental para que açóes efetivas de controle possam ser direcionadas.

Nos estudos etiológicos, abordagens de base individual ou com dados agregados são complementares e evidenciam distintos aspectos do processo de saúde-doença, ajudando a entender a múltipla trama de fatores que modificam o perfil de distribuição do câncer entre as classes sociais. Para a obtenção de estudos robustos, nota-se a tendência crescente da condução de estudos multicêntricos com pesquisadores organizados em redes. Isto permite obter amplas amostras, com maior grau de heterogeneidade em relação aos fatores de risco estudados e, conseqüentemente, maior flexibilidade para explorações analíticas. $\mathrm{Na}$ análise epidemiológica, a escolha preferencial deve recair sobre os modelos hierárquicos multiníveis, pois permitem estabelecer uma articulação entre os fatores de risco em diferentes níveis de determinação para examinar desfechos de interesse relacionados ao câncer (BENTLEY et al., 2008).

As pesquisas epidemiológicas em câncer não devem permanecer restritas ao período da vida adulta, próximo à manifestação da doença. Como já comentado, o período de latência em câncer é longo e, em geral, a exposição aos fatores causais iniciadores está longínqua do momento do diagnóstico. Estudos da exposição aos fatores de risco ao longo do curso da vida são úteis para uma visão mais abrangente da etiologia do câncer. Exposições a cancerígenos na vida intra-uterina ou na infância precoce têm sido implicadas na ocorrência de câncer na vida adulta (BAIK et al., 2005; BARTON et al., 2005; FORMAN et al., 2005). Muitas das situações de vulnerabilidade nas fases iniciais da vida estão socialmente determinadas (UAUY; SOLOMONS, 2005).

Do ponto de vista de políticas públicas em saúde, reduzir as disparidades sociais tem ampla repercussão para a população, pois os benefícios se estendem além dos grupos socialmente vulneráveis, como assinalado por Rose (1985; 1992), ao discutir o paradoxo do princípio da prevenção. Nos Estados Unidos, estabeleceu-se a meta de eliminar até 2010 as disparidades em saúde de acordo com raça ou etnia, educação ou renda, deficiência física, território geográfico ou orientação sexual (US DEPARTMENT..., 2000). Todavia, são grandes os desafios metodológicos 
para o acompanhamento dessas políticas. Um aspecto relevante diz respeito às próprias medidas para avaliar e monitorar as diferenças sociais e seu impacto sobre a ocorrência de câncer (HARPER et al., 2008). Para os países em desenvolvimento, adicionalmente, há ainda a sempre presente questão da qualidade dos dados, que pode inviabilizar o seguimento adequado das metas a serem atingidas.

O declínio da mortalidade por tumores de mama e de colo de útero está, em grande parte, relacionado a políticas de rastreamento. Nos países em desenvolvimento, essas açôes apresentam entraves que impedem efeitos relevantes, a exemplo do relatado sobre o programa de rastreamento do câncer de colo uterino no México (LAZCANOPONCE et al., 1997; HERNANDÉZ-AVILA et al., 1998). Portanto, há necessidade de serem estudados os meios para superar tais dificuldades. Múltiplas estratégias de prevenção secundária para o controle do câncer de colo de útero dentro de um país podem ser a alternativa, considerando-se as particularidades regionais, pois algumas ações parecem ter efetividade local, mas não global (GAKIDOU et al., 2008). O planejamento das açóes de rastreamento deve estar cientificamente alicerçado, sob pena do uso inadequado de recursos humanos e financeiros, como foi observado no programa de rastreamento de câncer de boca no Estado de São Paulo, vinculado à vacinação contra a gripe em idosos (ANTUNES et al., 2007).

Reconhecer as disparidades em saúde contribui para a formulação de políticas de redistribuição objetivando a promoção do bem-estar e de justiça social. Estudos sugerem que a carga do câncer, pelo menos em parte e no longo prazo, será reduzida pela diminuição das disparidades sociais em relação ao acesso à prevenção primária, ao diagnóstico precoce, a tratamentos padronizados e à reabilitação.

\section{Referências}

ALMEIDA FILHO, Neomar et al. Alcohol drinking patterns by gender, ethnicity, and social class in Bahia, Brazil. Revista de Saúde Pública, São Paulo, v. 38, n.1, p. 45-54, feb. 2004.

ANTUNES, José Leopoldo Ferreira; TOPORCOV, Tatiana Natasha; WÜNSCH FILHO, Victor. Resolutividade da campanha de prevenção e diagnóstico precoce do câncer bucal em São Paulo, Brasil. Revista Panamericana de Salud Publica, v. 21, n.1, p. 30-36, jan 2007.

BAIK, Inkyung et al. Association of fetal hormone levels with stem cell potential: evidence for early life roots of human cancer. Cancer Research, v. 65, p. 358-363, jan. 2005.

BALLARD-BARBASH, Rachel et al. Obesity and body composition. In: Schottenfeld David, Fraumeni Jr Joseph F. Cancer epidemiology and prevention. New York: Oxford University Press, aug. 2006. 
BARTEN, F et al. Contextualising worker's health and safety in urban settings: the need for a global perspective and an integrated approach. Habitat International, v. 32, p. 223-236, 2008.

BARTON, Hugh A. et al. Assessing susceptibility from early-life exposure to carcinogens. Environmental Health Perspect, v. 113, n.9, p. 1125-1133, sept. 2005.

BENTLEY, Rebecca et al. Area disadvantage, individual socio-economic position, and premature cancer mortality in Australia 1998 to 2000: a multilevel analysis. Cancer Causes and Control, v. 19, n.2, p. 183-193, mar. 2008.

BLEICH, Sara. et al. Why is the developed world obese? Annual Review of Public Health, v. 29, p. 273-295, apr. 2008.

BLOOMFIELD, Kim et al. Social inequalities in alcohol consumption and alcohol-related problems in the study countries of the EU concerted action "Gender, Culture and Alcohol Problems: a Multi-national Study". Alcohol and Alcoholism, [New York]: Oxford University Press, v. 41, p. 2636, oct. 2006. Supplement 1.

BOFFETTA, P. et al. Exposure to occupational carcinogens and social class differences in cancer occurrence. IARC Scientific Publication, v. 138, p. 331-341, 2007.

BRASIL. Ministério da Saúde. Instituto Nacional de Alimentação e Nutrição (INAN). Pesquisa nacional sobre saúde e nutrição: perfil de crescimento da população brasileira de 0 a 25 anos. INAN: Brasília, 1990.

BRASIL. Ministério da Saúde. Secretaria de Atenção à Saúde. Instituto Nacional do Câncer. Coordenação de Prevenção e Vigilância de Câncer. Estimativas 2008: incidência de câncer no Brasil. Rio de Janeiro: INCA, 2007.

CARVALHO, André L. et al. Cancer of the oral cavity: a comparison between institutions in a developing and a developed nation. Head Neck, New York, v. 26, n.1, p. 31-38, 2004.

CÉSAR, Chester Luiz Galvao et al. Saúde e condição de vida em São Paulo. São Paulo: USP/ FSP, 2005. 212 p.

CHRISTIANI, D.C.; WANG, X.R. Impact of chemical and physical exposures on worker's health In: HEYMANN, Jody. Global inequalities at work: work's impact on the health of individuals, families and societies. New York: Oxford University Press, 2003. p. 15-30

CONWAY, David I. et al. Socioeconomic inequalities and oral cancer risk: a systematic review and metaanalysis of case-control studies. International Journal of Cancer, v. 122, p. 2811-2819, jun. 2008.

COSTA, Juvenal. S. et al. Consumo abusivo de álcool e fatores associados: estudo de base populacional. Revista de Saúde Pública, São Paulo, v. 38, p. 284-291, abr. 2004.

CURADO, M. P. et al (Ed.). Cancer Incidence in five continents, v. IX. IARC Scientific Publications, v. $160,2007$.

ELUF NETO, José; WÜNSCH FILHO, Victor. Screening faz bem à saúde? Revista da Associação Médica Brasileira, v. 46, p. 310-311, out/dez 2004. 
FAGGIANO, F. et al. Socioeconomic differences in cancer incidence and mortality. IARC Scientific Public, v. 138, p. 65-176, 1997.

FEDERICO, B. et al. Trends in educational inequalities in smoking in northern, mid and southern Italy, 1980-2000. Preventive Medicine, v. 39, n.5, p. 919-926, 2004.

FORMAN, Michele R. et al. Through the looking glass at early-life exposures and breast cancer risk. Cancer Investigation, v. 23, n.7, p. 609-624, 2005.

FRIEL, Sharon.; CHOPRA, Mickey.; SATCHER, David. Unequal weight: equity oriented policy responses to the global obesity epidemic. BJM, v. 335, p. 1 241-1 243, dec. 2007.

FUKUDA, Y.; NAKAMURA, K.; TAKANO, T. Socioeconomic pattern of smoking in Japan: income inequality and gender and age differences. Annals of Epidemiology, v. 5, p.365-372, 2005.

GAKIDOU, E.; NORDHAGEN, S.; OBERMEYER, Z. Coverage of cervical cancer screening in 57 countries: low average levels and large inequalities. Plos Medicine, v. 5, p. 863-868, 2008.

GALEA, Sandro et al. Education inequality and use of cigarettes, alcohol, and marijuana. Drug and Alcohol Dependence, v. 90, p. S4-S15, sept. 2007.

HARMAN, Juliet et al. Socioeconomic gradients in smoking among young women: A British survey. Social Science and Medicine, v. 63, n.11, p. 2791-2800, 2006.

HARPER, Sam. et al. An overview of methods for monitoring social disparities in cancer with an example using trends in lung cancer incidence by area-socioeconomic position and race-etnicity, 1992-2004. American Journal of Epidemiology, v. 67, n.8, p.889-899, 2008.

HERNÁNDEZ-AVILA, Maurício et al. Evaluation of the cervical cancer screening programme in Mexico: a population-based case-control study. International Journal of Epidemiology, v. 27, p. 370-376, june. 1998.

HULSHOF, K. F. Socio-economic status, dietary intake and 10 years trends: the Dutch National Food Consumption Survey. European Journal of Clinical Nutritional, v. 57, p. 128-137, 2003.

IGLESIAS, Roberto et al. Controle do tabagismo no Brasil. Washington: World Bank, 2007.

BRASIL. Ministério da Saúde. Secretaria de Vigilância em Saúde. Secretaria de Atenção a Saúde. Instituto Nacional do Câncer. Inquérito domiciliar sobre comportamentos de risco e morbidade referida de doenças e agravos não transmissiveis:Brasil, 15 capitais e Distrito Federal, 2002-2003. Rio de Janeiro: INCA, Coordenação de Prevenção e Vigilância, 2004.

JAIME, Patricia Constance; MONTEIRO, Carlos Augusto. Fruit and vegetable intake by Brazilian adults, 2003. Cadernos de Saúde Pública, Rio de Janeiro, v. 21, p. S19-S24, 2005. Supplement 1. JHA, P.; CHALOUPKA, F.J. Curbing the epidemic: governments and the economics of tobacco control. Washington: World Bank, 1999.

JUÁREZ-FIGUEROA, Luis A. et al. Human Papillomavirus: a highly prevalent sexually 
transmitted disease agent among female sex workers from Mexico City. Sexually Transmitted Diseases, v. 28, n.3, p. 125-130, 2001.

KAPLAN G.A. The role of epidemiologists in eradicability of poverty. Lancet , v. 352, p. 16271628, sept. 1998.

KAWACHI, Ichiro; KROENKE, Candyce. Socioeconomic disparities in cancer incidence and mortality. New York: Oxford University Press, p. 174-188, 2006.

KHANG, Young-Ho; CHO, Hong-Jun. Socioeconomic inequality in cigarette smoking: trends by gender, age, and socioeconomic position in South Korea, 1989-2003. Preventive Medicine, v. 42, n.6, p. 415-422, jun. 2006.

KIVIMÄKI, Mika et al. Temporary employment and risk of overall and cause-especific mortality. American Journal of Epidemiology, v. 158, n.7, p. 663-668, 2003.

KUEHNI, Claudia; ZWAHLEN, Marcel. Numerous, heterogeneous and often poor: the studies on childhood leukemia and socioeconomic status. International Journal of Epidemiology, v 35, p. 384-385, 2006.

LAZCANO-PONCE, Eduardo Cesar et al. The cervical cancer screening program in Mexico: problems with access and coverage. Cancer Causes Control, v. 8, n.8, p. 698-704, sept. 1997.

LEVY-COSTA, Renata Bertazzi et al. Disponibilidade domiciliar de alimentos no Brasil: distribuição e evolução (1974-2003). Revista de Saúde Pública, São Paulo, v. 39, n.4, p. 530-540, aug. 2005.

LIMA-COSTA, Maria Fernanda. A escolaridade afeta igualmente comportamentos prejudiciais à saúde de idosos e adultos mais jovens?: inquérito de saúde da Região Metropolitana de Belo Horizonte, Minas Gerais, Brasil. Epidemiologia e Serviços de Saúde, Brasília, v. 13, n.4, p. 201-208, dez. 2004.

LOLIO, Cecília Amaro de et al. Hipertensão arterial e possíveis fatores de risco. Revista de Saúde Pública, São Paulo, v. 27, n.5, p. 357-362, out.1993.

LOPEZ, Diana M. et al. Making cancer health disparities history: Report of the Trans-HHS Cancer Health Disparities Progress Review Group. Washington: U.S. Department of Health and Human Services, mar. 2004.

LOSTAO, Lourdes et al. Patient cost sharing and physician visits by socioeconomic position: finding in three Western European countries. Journal of Epidemiology and Community Health, v. 61, p. 416-420, may. 2007.

LYNGE, Elsebeth et al. Desigualdades sociales y cáncer. In: - Prevención del cáncer: estrategias basadas en la evidencia. Ginebra: UICC, Unión Internacional Contra el Cáncer. 2006. p. 29-47. MALTA, Deborah Carvalho et al. Tendências de mortalidade do câncer de pulmão, traquéia e brônquios no Brasil, 1980-2003. Jornal Brasileiro de Pneumologia, v. 33, p. 536-543, set/out. 2007. MARSHALL, James R.; FREUDENHEIM, Jo. Alcohol. In: Schottenfeld David, 
Fraumeni Jr, Joseph F. Cancer epidemiology and prevention. 3th ed. New York: Oxford University Press, 2006. p. 243-258.

MATHERS, Colin D. et al. Counting the dead and what they died from: an assessment of the global status of cause of death data. Bulletin of the World Health Organization, v. 83, n.3, p. 171177 , mar. 2005.

MELCHIOR, Maria et al. Occupational class, occupational mobility and cancer incidence among midle-aged men and women: a prospective study of the French Gazel Study. Cancer Causes Control, v. 16, p. 515-524, 2004.

MENDONZA-SASSI, Raul A.; BERIA, Jorge U. Prevalence of alcohol use disorders and associated factors: a population-based study using AUDIT in southern Brazil. Addiction, v. 98, n.6, p. 799-804, 2003.

MENVIELLE, Gwenn. et al. Smoking alcohol drinking, occupational exposures and social inequalities in hypopharyngeal and laryngeal cancer. International Journal of Epidemiology, v. 33, n.4, p. 799-806, 2004.

MINAKER, Leia M. et al. School region socio-economic status and geographic locale is associated with food behaviour of Ontário and Alberta adolescents. Canadian Journal of Public Health, v. 97 , n.5, p. 357-361, sept/oct. 2006.

MONTEIRO, Carlos Augusto et al. Obesity and inequalities in health in the developing world. International Journal of Obesity and Related Metabolic Disorders, v. 28, p. 1181-1186, 2004.

MONTEIRO, Carlos A.; CONDE, Wolney L.; POPKIN, Barry M. Income-specific trends in obesity in Brazil: 1975-2003. American Journal of Public Health, v. 97, n.10, p. 1808-1812, oct. 2007.

MOREIRA, Leila Beltrami et al. Alcoholic beverage consumption and associated factors in Porto Alegre, a southern Brazilian city: a population-based survey. Journal of Studies on Alcohol, v. 57, n.3, p. 253-259, may. 1996.

MOREIRA, Leila Beltrami et al. Prevalência de tabagismo e fatores associados em área metropolitana da região sul do Brasil. Revista de Saúde Pública, São Paulo, v. 29, n.1, p. 46-51, fev. 1995.

Organization for Economic Co-operation and Development. Guiding principles for chemical accident prevention, preparedness and response. Guidance for industry (including management and labour), public authorities, communities and other stakeholders, 2003. Disponivel em:<http://www2.oecd.org/ guidingprinciples/document/pg013.asp>. Acesso em: 27 abr. 2008.

PALACIO-MEJÍA, Lina Sofia et al. Cervical cancer, a disease of poverty: mortality differences between urban and rural areas in Mexico. Salud Pública de México, v. 45, p. 315-325, 2003. Suppl. 3.

PARKIN, D. Maxwell et al (Ed.). Cancer Incidence in five continents, v. Vlll. IARC Scientific Publications, v. 155, p. 1-782, 2002.

PEARCE, Neil et al. Industrialization and health. IARC Scientific Publiations, v. 127, p. 7-22, 1994. 
PERCY, Constance.; STANEK, Edward.; GLOECKLER, Lynn. Accuracy of cancer death certificates and its effect on cancer mortality statistics. American Journal of Public Health, v. 15, p. 115-123, 1981.

PHAROAH, P.D. Genetic susceptibility, predicting risk and preventing cancer. Recent Results in Cancer Research, v. 163, p.7-18, 2003.

POOLE, C. et al. Socioeconomic status and childhood leukaemia: a review. International Journal of Epidemiology, v. 35, n. 2, p. 370-384, apr. 2006.

PRADO, Mariângela Cainelli de Oliveira. Câncer feminino: evolução da mortalidade por cancer de mama e do colo de útero em distritos do município de São Paulo, 1985-1999. Tese (Doutorado)Faculdade de Saúde Pública da USP, São Paulo, 2002.

ROSE, Geoffrey. Sick individuals and sick populations. International Journal of Epidemiology, v. 14, n.1, p. 32-38, 1985.

ROSE, Geoffrey. The strategy of preventive medicine. New York: Oxford University Press, 1992.

SANKARANARAYANAN; BLACK, R. J.; PARKIN, D. M. Cancer survival in developing countries. IARC Scientific Publications, v. 145, 1998.

SANKARANARAYANAN, Rengaswamy et al. Oral Cancer Screening Study Group. Effect of screening on oral cancer mortality in Kerala, India: a cluster-randomized controlled trial. Lancet, v. 365, p. 1927-33, 2005.

SANTO, Augusto H. Causas mal definidas de morte e óbitos sem assistência. Revista da Associação Médica Brasileira, v. 54, n.1, p. 23-28, jan/feb 2008.

SCHOEN, C.; DOTY, M. M. Inequities in access to medical care in five countries, findings from the 2001 Commonwealth Fund International Health Policy Survey. Health Policy, v. 67, n.3, p. 309-322, mar. 2004.

RIO GRANDE DO SUL. Secretaria do Planejamento e Gestão. Departamento de Planejamento Governamental. Atlas Socioeconômico do Rio Grande do Sul. Disponível em: <http://www.rs.gov.br/ atlas/>. Acesso em: 29 de abril de 2008.

SHAHAR, Danit et al. Diet and eating habits in high and low socioeconomic groups. Nutrition, v. 21 , n. 5, p. 559-566, may. 2005.

SILVI, John. On the estimation of mortality rates for countries of the Americas. Epidemiological Bulletin, v. 24, n.4, p. 1-5, dec. 2003.

SOTERIADES, Elpidoforos S.; DIFRANZA, Joseph R. Parent's socioeconomic status, adolescents' disposable income, and adolescents' smoking status in Massachusetts. American Journal of Public Health, v. 93, n.7, p. 1155-1160, july. 2003.

SUBRAMANIAN, S. V. et al. Role of socioeconomic markers and state prohibition policy in 
predicting alcohol consumption among men and women in India: a multilevel statistical analysis. Bulletin of the World Health Organizartion, v. 83, n.11, p. 829-836, 2005.

SZWARCWALD, Célia Iandmann et al. Pesquisa mundial de saúde: o Brasil em números. RADIS, v. 23, p. 14-33, 2004.

TRAVASSOS, Claudia et al. Utilização dos serviços de saúde no Brasil: gênero, características familiares e condição social. Revista Panamericana de Salud Publica, v. 11, n.5-6, p. 365-373, may/june. 2002. UAUY, R.; MONTEIRO, C.A. The challenge of improving food and nutrition in Latin America. Food and Nutrition Bulletin, v. 25, n.5, p. 175-182, jun. 2004.

UAUY, Ricardo.; SOLOMONS, Noel. Diet, nutrition, and life-course approach to cancer prevention. The Journal of Nutrition, v. 135, n.12S, p. 2934S-2945S, dec. 2005.

UNITED STATES. Department of Health and Human Services. Health people 2010: understanding and improving health. Washington, DC: US Government Printing Office, 2000. VAN OERS, J. A. et al. Alcohol consumption, alcohol-related problems, problem drinking, and socioeconomic status. Alcohol and Alcoholism, v. 34, n.1, p. 78-88, jan/feb.1999.

VEGLIA, Fabrizio et al. Occupational exposures, environmental tobacco smoke, and lung cancer. Epidemiology, v. 18, n.6, p. 769-775, nov. 2007.

VICTORA, C.G. et al. The role of conceptual frameworks in epidemiological analysis: a hierarchical approach. International Journal of Epidemiology, v. 26, n.1, p. 224-227, feb. 1997.

VILLALBI, J. R. et al. Changing epidemiology of smoking: Barcelona 2000-2001. Revista Clínica Española, v. 204, n.6, p. 312-316, jun. 2004.

WEN, C. P. et al. Smoking behaviour in Taiwan, 2001. Tobacco Control, v. 14, p. i51-i55, june. 2005.

WHITLOCK, G.et al. Socioeconomic distribution of smoking in a population of $10,529 \mathrm{New}$ Zealanders. The New Zealand Medical Journal, v. 110, p. 327-330, 1997.

WÜNSCH FILHO, Victor; MONCAU, José Eduardo. Mortalidade por câncer no Brasil 19801995: padrões regionais e tendências temporais. Revista da Associação Medica Brasileira, São Paulo, v. 48, n.3, p. 250-257, jul/set. 2002. 


\section{Prospects of research on social determinants in cancer}

Socioeconomic differences have effects on epidemiological profile of cancer as regards incidence, mortality, survival and quality of life after diagnosis. In this study are examined the inequalities of cancer occurrence in the Brazilian population and summarized the evidences of epidemiological research on social determinants in cancer. The main factors that modulate the influence of socioeconomic conditions in cancer are considered, such as smoking, alcohol consumption, diet and obesity, occupation, and access to health services. Modifications of social conditions depend on broader changes in society, as improvements of educational level; however, well-conducted epidemiological investigations can instruct the planning of interventions aimed at reducing the impact of social determinants on cancer. These studies should focus on methods to improve the quality of information on incidence and mortality; regular population surveys on the prevalence of cancer risk factors; study designs to examine cancer etiological factors in its relations with social status; implementation and assessment of effectiveness of screening programs for early detection of tumors; and evaluation of access to diagnosis and treatment. These researches should include populations in different regions of the world, particularly those living in places that lack the benefits provided by the dynamics of the present global economic system.

> Key words: cancer; socioeconomic conditions; inequalities in health. 